\title{
Outcome of computer-assisted surgery in patients with chronic rhinosinusitis
}

\author{
S A Mueller, M CAVERSACCiO*
}

\begin{abstract}
Objective: To compare the complication rates and outcome of computer-assisted versus non-computer-assisted functional endoscopic sinus surgery.

Methods: We reviewed retrospectively the medical records of 276 patients who had undergone sinus surgery for chronic rhinosinusitis with $(n=108)$ or without $(n=168)$ computer assistance, from 1996 to 2004 , to determine the incidence of complications and need for revision surgery.

Results: The incidence of complications was 6.5 per cent in the computer-assisted group and 6.0 per cent in the non-computer-assisted group $(p=1.00)$. In the computer-assisted group, 9.2 per cent needed revision surgery, compared with 10.7 per cent in the non-assisted group $(p=0.84)$.

Conclusions: Although our study found no significant difference in complications or revision rates, computer-assisted surgery serves as an important orientation aid during functional endoscopic sinus surgery.
\end{abstract}

Key words: Surgery, Computer-Assisted; Endoscopy; Sinusitis; Intraoperative Complications

\section{Introduction}

The use of computer assistance during functional endoscopic sinus surgery (FESS) has expanded rapidly since its introduction in the 1990s. ${ }^{1-3}$ Due to ever-advancing technical improvements in safety, accuracy and manageability, computer-assisted FESS has become an indispensable tool in ENT and neurosurgery. In paranasal sinus surgery, computer-assisted surgery has been shown to enhance surgeons' confidence, ${ }^{4}$ especially in cases in which anatomical landmarks have been altered iatrogenically or through extensive disease. ${ }^{5}$ The technique's usefulness in the treatment of sinus disease has been demonstrated. ${ }^{6,7}$ However, it remains unclear whether computer-assisted FESS reduces the incidence of such typical surgical complications as orbital trauma, bleeding, direct brain trauma and cerebrospinal fluid (CSF) leakage, compared with non-image-guided FESS. 8,9

The most commonly mentioned drawbacks of computer-assisted surgery are increased cost and increased time demands (during both installation and the surgery itself). ${ }^{4,10,11}$ In addition, the computer-assisted surgical systems in use today have an accuracy of 0.5 to $2 \mathrm{~mm}^{8,12-14}$ This slight inaccuracy, potentially compounded by errors in image generation and referencing procedures, implies that, although it may serve as an affirming guide, computer-assisted surgery cannot replace a combination of surgical experience and thorough knowledge of the complex paranasal sinus anatomy. ${ }^{12}$ On the other hand, computer-assisted surgical systems have proved their worth as educational tools for surgical training. ${ }^{15,16}$

Today, computer-assisted surgery is used for a variety of diseases, in a variety of settings, including endoscopic, macroscopic and microscopic surgery.

One of the most frequent indications for surgery of the paranasal sinuses is chronic rhinosinusitis. According to the 'European Position Paper on Rhinosinusitis and Nasal Polyps, ${ }^{17}$ the prevalence of chronic rhinosinusitis is between 2 and 6 per cent, being higher in females and increasing with age. Predisposing factors for chronic rhinosinusitis include primary and secondary ciliary dyskinesia, cystic fibrosis, allergic asthma, primary and secondary immunodeficiency, and local factors such as anatomical variation. Furthermore, diseases involving the dental roots may have long term effects on the maxillary sinuses. Increased usage of FESS correlates with an increased incidence of mucoceles. Nasal polyps are considered a subgroup of chronic rhinosinusitis, and an association with aspirin sensitivity has been reported.

The current medical treatment concept for chronic rhinosinusitis and nasal polyposis draws upon

From the Department of General Surgery, Spital Oberengadin, Samedan, and the *University Clinic of ENT, Head and Neck Surgery, Inselspital Berne, Switzerland.

Accepted for publication: 3 September 2009. First published online 11 December 2009. 
multiple approaches. Apart from saline washes to remove secretions, irritants and allergens, both topical and systemic glucocorticoids are used to inhibit inflammation, as are antileukotriene agents such as Montelukast. Antimicrobials are administered intermittently in cases of bacterial or fungal superinfection, but the long term use of antimicrobials remains controversial. Surgery is indicated in the management of chronic rhinosinusitis and nasal polyposis only after conservative measures have failed to produce lasting improvement in symptoms. The aim of surgical intervention is to enhance ventilation of the affected sinuses by removing elements that may cause sinus congestion.

Computer-assisted surgery has been in use at the University Clinic of ENT, Head and Neck Surgery, Inselspital Berne, Switzerland for more than 10 years. This study aimed to evaluate our experience of computer-assisted surgery, regarding complications and outcome in patients treated specifically for chronic rhinosinusitis.

\section{Materials and methods}

Study design

Data were collected in a retrospective review of the medical records of 276 patients diagnosed with chronic rhinosinusitis or nasal polyps and undergoing paranasal sinus surgery at the University Clinic of ENT, Head and Neck Surgery, Inselspital Berne, Switzerland between January 1996 and December 2004.

The primary aim of the study was to compare the complication rates of computer-assisted FESS versus non-computer-assisted FESS. Only complications related directly to the surgical procedure were considered; complications due to anaesthesia or hospitalisation (not directly associated with surgery) were excluded. Possible major complications included: bleeding from a major vessel (i.e. anterior ethmoidal artery, sphenopalatine artery or internal carotid artery) and/or the need for transfusion; orbital trauma; optical nerve damage; intracranial trauma (i.e. brain abscess, meningitis, intracranial haemorrhage or direct brain trauma); CSF leakage; and anosmia. Possible minor complications included: diffuse bleeding without the need for transfusion; perforation of the lamina papyracea without periorbital damage; ecchymosis of the eyelid; and hyposmia. Data were collected from surgical notes and hospital medical reports.

The secondary aim of the study was to compare the two surgical techniques regarding relapse rates and the need for revision surgery during the documented follow-up period.

\section{Patients}

Male and female patients over the age of 16 years who had been treated surgically for chronic rhinosinusitis or nasal polyps during the study period were included in the study. Patients with cystic fibrosis, gross immunodeficiency, primary ciliary dyskinesia, systemic vasculitis or granulomatous diseases were excluded, as were those with chronic rhinosinusitis of dental or fungal origin. Furthermore, only patients treated with paranasal surgery using an endonasal approach were included.

Using these selection criteria, 276 patients were selected, of which 108 received computer-assisted FESS while the remaining 168 received noncomputed-assisted FESS. Use of computer-assisted FESS in the individual patient depended on whether the treating surgeon was appropriately trained in the computer-assisted surgery technique. In all patients, the Lund-McKay score ${ }^{18}$ was used to stage the disease on computed tomography (CT), and in some cases magnetic resonance imaging (MRI), prior to surgery. This score separately rates the condition of the anterior ethmoidal, posterior ethmoidal, maxillary, frontal and sphenoidal sinuses as well the ostiomeatal complex, awarding from zero to two points, leading to a total bilateral score ranging from zero to 24 points.

\section{Surgery}

All procedures were performed at the University Clinic of ENT, Head and Neck Surgery, Inselspital Berne, Switzerland, by various experienced surgeons. All but one patient received general anaesthesia. The one exception received local anaesthesia because of severe cardiomyopathy. The surgical procedures were performed according to previously described techniques, and included infundibulotomy, maxillary antrostomy, anterior and/or posterior ethmoidectomy, and sphenoidectomy, with or without enlargement of the frontal recess (type one to three according to Draf).

\section{Technical aspects}

For computer-assisted surgery, we used a frameless navigation system developed at the Maurice E Müller Institute for Biomechanics in Bern, in collaboration with the University Clinic of ENT, Head and Neck Surgery, Inselspital Berne, Switzerland and Medivision (Synthes-Stratec Medical, Solothurn, Switzerland). The SurgiGATE ORL system contains an infrared-based, three-dimensional tracking facility (Optotrack 3020; Northern Digital, Waterloo, Ontario, Canada) which accurately determines the position of infrared light emitting diodes. Four infrared light emitting diodes placed on each instrument enable localisation of both the instrument tip and its axis, with an accuracy of $0.1 \mathrm{~mm}$ in the $x$ and $y$ axes and $1.5 \mathrm{~mm}$ in the $z$ axis. ${ }^{19}$ To permit free head movement during surgery, a dynamic reference base carrying four infrared light emitting diodes is positioned either on the upper jaw (on an imprint of the patient's dentition) or fixed to the skull with screws. The exact position of the head, in relation to previously obtained CT or MRI data, is ascertained by pair-point matching, using a needle pointer. Using this method, a clinical accuracy of 0.5 to $2.0 \mathrm{~mm}$ is achieved. All instruments were calibrated before each surgical intervention. 


\section{Statistics}

All statistical analyses were performed using Graphpad InStat version 3 software (Graphpad Software, La Jolla, California, USA). Complications were defined as nominal data and compared by means of Fisher's exact test. Ordinal data (such as disease severity, disease relapse and revision surgery) were analysed using the Mann-Whitney $U$ test. The unpaired z-test was used for rational data such as age. In all analyses, a $p$ value of less than 0.05 was considered significant.

\section{Results and analysis}

A total of 276 patients, all diagnosed with chronic rhinosinusitis or nasal polyps, received surgical treatment during the study period, of whom 108 underwent computer-assisted FESS and 168 non-computerassisted FESS. In both groups, there was a predominance of men: the computer-assisted surgery group comprised 69 (63.9 per cent) males and 39 (36.1 per cent) females, and the non-computer-assisted group 109 (64.9 per cent) males and 59 (35.1 per cent) females. Mean age at the time of surgery was significantly higher in the computer-assisted group, at 48.4 years (range 17.6-82.1 years), than in the non-computer-assisted group, at 42.3 years (range $16.3-83.5$ years $)(p=0.0007)$.

Chronic rhinosinusitis was diagnosed in 129 patients and nasal polyps in 147 (53.3 per cent). Asthma was present in 70 patients $(25.4$ per cent) and allergic rhinitis in 58 (21.0 per cent). Other frequent comorbidities included deformation of the nasal septum $(n=47 ; 17.0$ per cent $)$ and mucocele (16; 5.8 per cent). The incidence of these comorbidities was similar in both groups. Disease extent was assessed by the Lund-McKay score; the computerassisted group had a mean bilateral score of 14.3 (median 14.0; range 3-24), which differed significantly from the non-computer-assisted group (mean bilateral score 10.0; median 9.0 ; range $1-24)(p<$ $0.0001)$. The mean documented follow-up time was 18.4 months (median 6.2 months; range 5 days to 9.7 years) in the computer-assisted group and 15.8 months (median 4.1 months; range 5 days to 10.5 years) in the non-computer-assisted group $(p=0.12)$.

Table I lists the incidence of major and minor complications.

In the computer-assisted surgery group, seven patients (6.5 per cent) suffered complications, of which three (2.7 per cent) were considered major and four (3.7 per cent) minor. In the non-computer-assisted group, 10 patients ( 6.0 per cent) suffered complications, three (1.8 per cent) major and seven ( 4.2 per cent) minor. The nature of the complications is shown in Table I. No significant difference in complication rates was found between the two groups, with $p=$ 0.68 for major complications and $p=1.00$ for minor complications and for all complications. Notably, major and minor orbital complications and skull base injuries were only observed in the non-computerassisted group. In the computer-assisted group, two instances of major bleeding occurred: the anterior
TABLE I

MAJOR AND MINOR SURGICAL COMPLICATIONS AFTER COMPUTER-ASSISTED AND NON-COMPUTER-ASSISTED FESS

\begin{tabular}{llr}
\hline Complication & $\begin{array}{c}\text { CA-FESS } \\
(n(\%))\end{array}$ & $\begin{array}{c}\text { FESS } \\
(n(\%))\end{array}$ \\
\hline Major & & \\
Major bleeding & $2(1.9)$ & $0(0)$ \\
Diplopia & $0(0)$ & $1(0.6)$ \\
Skull base injury & $0(0)$ & $1(0.6)$ \\
Anosmia & $1(0.9)$ & $1(0.6)$ \\
Total & $3(2.8)$ & $3(1.8)$ \\
Minor & & \\
Diffuse bleeding, no transfusion needed & $4(3.7)$ & $5(3.0)$ \\
Lamina papyracea perforation & $0(0)$ & $2(1.2)$ \\
Total & $4(3.7)$ & $7(4.2)$ \\
Total, all complications & $7(6.5)$ & $10(6.0)$ \\
\hline
\end{tabular}

$\mathrm{CA}=$ computer-assisted; FESS $=$ functional endoscopic sinus surgery

ethmoidal artery was affected in one case, while the origin of bleeding could not be clearly identified in the other.

Revision surgery was performed in 10 (9.2 per cent) patients in the computer-assisted group and 18 (10.7 per cent) in the non-computer-assisted group $(p=0.84)$.

\section{Discussion}

Endoscopic sinus surgery is frequently performed by otolaryngologists, and is the treatment of choice for various illnesses of the paranasal sinuses and skull base. In the last decade, traditional endoscopy has been complemented by computer-assisted surgery, providing an additional orientation guide. Computerassisted surgery has become a widespread tool in surgery of the paranasal sinuses and other areas such as the skull base and the orbit. It has proved its usefulness as a training medium, and the additional, threedimensional depiction of the operative field is known to enhance surgeons' confidence.,16 Computerassisted surgery is especially useful in cases where orientation in the labyrinthine paranasal sinuses is additionally impeded. Such situations may occur in revision procedures or where extensive disease has destroyed anatomical landmarks. The question of whether computer-assisted surgery can be used routinely in all patients is complicated by the fact that it remains unclear whether surgeons' improved confidence results in a reduced incidence of complications. Furthermore, conclusive data on how computerassisted FESS influences outcome and patient's quality of life, compared with non-computer-assisted FESS, is still lacking. This is mostly due to the logistical difficulties inherent in recruiting and supervising a cohort large enough to yield significant results, and to the concomitant ethical concerns over randomisation.

To our knowledge, at present only a few studies have shown a statistically significant benefit of computer-assisted FESS over non-image-guided FESS. Fried et $a l^{20}$ found a lower complication rate in patients treated with computer-assisted versus non-computer-assisted surgery. Another study 
found that post-operative quality of life was superior in patients undergoing computer-assisted FESS versus non-image-guided FESS. ${ }^{21}$ However, this study only evaluated quality of life six months after surgery, and made no statement on long term quality of life. Many other studies have failed to find significant differences between the two techniques. Tabaee et al. ${ }^{22}$ conducted a retrospective study of 239 patients, and found no differences in the incidence of complications and revision surgery, or in quality of life. All surgical procedures were performed by a single surgeon. In our study, multiple otolaryngologists performed the procedures, thus raising the possibility that complex cases were mostly handled by more experienced surgeons. On the other hand, it is evident that a single surgeon's learning curve may affect the outcome of surgery, especially as the two groups in the study by Tabaee et $a .^{22}$ were treated sequentially. In a prospective study of 121 cases over 12 months, Tschopp and Thomaser ${ }^{9}$ detected no improvement in either quality of life or CT scan appearance, comparing patients treated with computer-assisted versus non-image-guided surgery.

The current, retrospective study is reported as an evaluation of our own experiences with computerassisted surgery, and as a contribution to the on-going discussion on the technique's application and safety. Multiple variables may be used to assess surgical outcome. Our study focused on the incidence of complications and the need for revision surgery. No statistically significant difference was detected in any of the variables assessed.

The incidence of complications in our study agrees with other reports. ${ }^{17,22}$ Interestingly, there was a trend towards a slightly lower major complication rate in the non-computer-assisted group (6.0 per cent, compared with 6.5 per cent in the computer-assisted group), although this difference was not statistically significant. This may be coincidence, but it must be considered that patients in the computer-assisted surgery group generally showed more extensive disease on pre-operative CT scans, with LundMcKay scores of 14.3 (versus 10.0 in the non-computer-assisted group; $p<0.0001)$. When comparing the nature of complications in the two groups, it is notable that complications in the computer-assisted group were limited to bleeding, while orbital complications (both major and minor) and skull base injury were only experienced in the non-computer-assisted group (see Table I). In this group, four of the 10 recorded complications affected the orbit, and these four cases represented 2.4 per cent of all non-image-guided surgical procedures. The structure that separates the orbit from the paranasal sinuses is the aptly named lamina papyracea. This very thin sheet of bone is one of the most vulnerable structures during surgical procedures involving the adjacent ethmoidal cells, and is easily perforated. Although identification of the lamina papyracea does not normally pose major problems, it is conceivable that, in cases with extensive mucosal swelling or bleeding, adequate orientation may be impaired. In this setting, the additional visualisation enabled by computer-assisted surgery, especially the exact depiction of osseous structures, may be crucial. This explanation may also be applicable to the possible lower incidence of skull base injuries seen with computerassisted surgery, as has been suggested by other authors. $^{22}$ However, larger studies are required to confirm this assumption. The one skull base injury recorded in this study was an osseous breach in the posterior margin of the sphenoid sinus, which was covered intra-operatively and caused no CSF leakage.

Intuitively, it would seem logical that the additional visual information made available by computer-assisted surgery would result in a lower incidence of complications. However, there are several factors capable of neutralising this apparent advantage. Firstly, the reported improvement in surgeons' confidence ${ }^{4}$ raises the risk of surgeons overestimating their capabilities and neglecting their training in non-image-guided surgery. Secondly, it is important to bear in mind that computer-assisted surgery does not offer a false feedback mechanism. Although the position of the instrument is accurately located by computer-assisted surgery, injuries to osseous structures or soft tissue do not appear in the three-dimensional depiction, as this would require continuous radiological monitoring during surgery. Even with computer-assisted surgery, a surgical mistake may therefore remain unnoticed if overlooked in the endoscopic view. Furthermore, limitations of accuracy and technical errors in image generation and referencing represent other sources of danger. It must therefore be stressed that computer-assisted surgery is not to be misused as a pilot, but only as a control tool complementing endoscopy. Likewise, computer-assisted surgery cannot replace meticulous examination of $\mathrm{CT}$ and MRI scans during surgical planning. Anatomical areas representing possible surgical difficulties must be recognised prior to surgery, in order to take adequate safety measures.

- In specialised clinics, computer-assisted surgery systems are commonly used during functional endoscopic sinus surgery

- Three-dimensional depiction of the operation site is a valuable orientation aid for surgeons

- However, computer-assisted surgical systems are costly and their outcome benefits unclear; thus, their routine use is questionable

- These authors recommend computer-assisted surgery be reserved for special settings and discourage its routine use, as no improvement in complication rates or outcome is evident

In our study, revision surgery was necessary in 9.2 per cent of the computer-assisted FESS patients, compared with 10.7 per cent of the non-computerassisted FESS patients $(p=0.84)$. Other studies have shown higher incidences of revision surgery in both groups. Senior et al. ${ }^{23}$ reported an 18 per cent rate of revision surgery after primary, non-image-guided 
FESS, whereas Tabaee et al. ${ }^{6}$ reported a 16.5 per cent rate after computer-assisted FESS. Revision surgery is carefully restricted at our institution, being performed only in cases of severe and persistent relapse unresponsive to long term conservative medical treatment. This may be one reason for the observed, comparatively low rate of revision surgery.

\section{Conclusion}

Although computer-assisted surgery systems may reassure surgeons of the position of their instruments and help to minimise intra-operative uncertainty, our study showed no significant improvement in complication rates or outcome, comparing computerassisted versus non-computer-assisted FESS. The routine use of computer-assisted surgical systems in all FESS patients seems unlikely to yield improved results, but raises the risk of dependence on the system due to neglect of training in non-image-guided FESS, and is therefore to be discouraged.

\section{Acknowledgements}

Marco Caversaccio is supported by the Swiss National Research Foundation for the Computer Aided and Image-Guided Medical Interventions project (www.co-me.ch).

\section{References}

1 Mosges R, Klimek L. Computer-assisted surgery of the paranasal sinuses. J Otolaryngol 1993;22:69-71

2 Anon JB, Lipman SP, Oppenheim D, Halt RA. Computerassisted endoscopic sinus surgery. Laryngoscope 1994;104: $901-5$

3 Anon JB, Klimek L, Mosges R, Zinreich J. Computerassisted endoscopic sinus surgery. An international review. Otolaryngol Clin North Am 1997;30:389-401

4 Metson RB, Cosenza MJ, Cunningham MJ, Randolph GW. Physician experience with an optical image guidance system for sinus surgery. Laryngoscope 2000;110:972-6

5 Metson R. Image-guided sinus surgery: lessons learned from the first 1000 cases. Otolaryngol Head Neck Surg 2003; 128:8-13

6 Tabaee A, Kacker A, Kassenoff TL, Anand V. Outcome of computer-assisted sinus surgery: a 5-year study. $\mathrm{Am} \mathrm{J}$ Rhinol 2003;17:291-7

7 Loehrl TA, Toohill RJ, Smith TL. Use of computer-aided surgery for frontal sinus ventilation. Laryngoscope 2000; 110:1962-7

8 Citardi MJ, Batra PS. Intraoperative surgical navigation for endoscopic sinus surgery: rationale and indications. Curr Opin Otolaryngol Head Neck Surg 2007;15:23-7

9 Tschopp KP, Thomaser EG. Outcome of functional endonasal sinus surgery with and without CT-navigation. Rhinology 2008;46:116-20
10 Gibbons MD, Gunn CG, Niwas S, Sillers MJ. Cost analysis of computer-aided endoscopic sinus surgery. Am J Rhinol 2001; 15:71-5

11 Caversaccio M, Freysinger W. Computer assistance for intraoperative navigation in ENT surgery. Minim Invasive Ther Allied Technol 2003;12:36-51

12 Grevers G, Menauer F, Leunig A, Caversaccio M, Kastenbauer E. Navigation surgery in diseases of the paranasal sinuses [in German]. Laryngorhinootologie 1999;78:41-6

13 Fried MP, Kleefield J, Gopal H, Reardon E, Ho BT, Kuhn FA. Image-guided endoscopic surgery: results of accuracy and performance in a multicenter clinical study using an electromagnetic tracking system. Laryngoscope 1997;107: 594-601

14 Knott PD, Batra PS, Butler RS, Citardi MJ. Contour and paired-point registration in a model for image-guided surgery. Laryngoscope 2006;116:1877-81

15 Casiano RR, Numa WA Jr. Efficacy of computed tomographic image-guided endoscopic sinus surgery in residency training programs. Laryngoscope 2000;110:1277-82

16 Caversaccio M, Zheng G, Nolte LP. Computer-aided surgery of the paranasal sinuses and the anterior skull base. HNO 2008;56:376-8, 780-2

17 European Academy of Allergology and Clinical Immunology. European position paper on rhinosinusitis and nasal polyps. Rhinol Suppl 2005;18:1-87

18 Lund VJ, Mackay IS. Staging in rhinosinusitis. Rhinology 1993;31:183-4

19 Caversaccio M, Bächler R, Lädrach K, Schroth G, Nolte LP, Häusler R. The "Bernese" frameless optical computer aided surgery system. Comput Aided Surg 1999;4:328-34

20 Fried MP, Moharir VM, Shin J, Taylor-Becker M, Morrison P. Comparison of endoscopis sinus surgery with and without image guidance. Am J Rhinol 2002;16:193-7

21 Javer AR, Genoway KA. Patient quality of life improvements with and without computer assistance in sinus surgery: outcomes study. J Otolaryngol 2006;35:373-9

22 Tabaee A, Hsu AK, Shrime MG, Rickert S, Close LG. Quality of life and complications following image-guided endoscopic sinus surgery. Otolaryngol Head Neck Surg 2006;135:76-80

23 Senior BA, Kennedy DW, Tanabodee J, Kroger H, Hassab $\mathrm{M}$, Lanza D. Long-term results of functional endoscopic sinus surgery. Laryngoscope 1998;108:151-7

Address for correspondence:

Dr Marco Caversaccio,

Deputy Chairman,

University Clinic of ENT,

Head and Neck Surgery,

Inselspital Berne,

Freiburgstrasse, 3010 Berne, Switzerland.

Fax: +41316324900

E-mail: marco.caversaccio@insel.ch

Dr M Caversaccio takes responsibility for the integrity

of the content of the paper.

Competing interests: None declared 